\title{
MODIFICAÇÃO DO TAMANHO DE NANOPARTÍCULAS POLIMÉRICAS POR COAGULAÇÃO CONTROLADA ATRAVÉS DA ADIÇÃO DE UM ALCANO DE BAIXO PONTO DE EBULIÇÃO
}

\author{
CLAUDIA A. CAPELETTO, CLAUDIA SAYER, PEDRO H. H. DE ARAÚJO \\ Universidade Federal de Santa Catarina, Departamento de Engenharia Química e Engenharia de \\ Alimentos \\ E-mail para contato: pedro@enq.ufsc.br
}

\begin{abstract}
RESUMO - O aumento controlado do tamanho de partícula de um látex polimérico previamente formado pode criar novas possibilidades de aplicação para produtos que não apresentam flexibilidade de obtenção em determinadas faixas de tamanho. Neste trabalho, látices de poliestireno com diferentes tamanhos de partículas e massas molares, obtidos via polimerização em emulsão, foram submetidos a um processo de coagulação controlada induzida pela presença de um alcano de baixo ponto de ebulição, que ao fim do processo pode ser removido e recuperado. O processo de coagulação foi realizado a $120^{\circ} \mathrm{C}$ por 180 minutos com teor de sólidos $0,5 \%$, seguido por um alívio de pressão realizado em temperaturas inferiores a $100^{\circ} \mathrm{C}$. Variáveis do processo, como teor de alcano e temperatura durante o alívio de pressão, também foram avaliadas. Os resultados mostram que partículas provenientes do látex com partículas maiores (diâmetro médio próximo de $240 \mathrm{~nm}$ ) não apresentaram aumento de tamanho significativo, enquanto que as partículas provenientes dos látices com partículas menores (diâmetro médio próximo de $60 \mathrm{~nm}$ ) apresentaram um aumento considerável (diâmetro médio final entre 800 e $1000 \mathrm{~nm}$ ), independente da massa molar, além de manterem a forma esférica.
\end{abstract}

\section{INTRODUÇÃO}

O tamanho e a distribuição de tamanho de partículas de um látex polimérico afeta a área de contato entre as partículas e o meio contínuo, reologia e opacidade do látex e, em última análise, a sua aplicação. Um considerável conjunto de diferentes técnicas de polimerização em meio disperso proporcionam a obtenção de uma gama ampla de tamanhos, com diâmetros indo de uns poucos nanômetros até milímetros. No entanto, para tamanhos contidos dentro da faixa de 0,5 à $20 \mu \mathrm{m}$, praticamente não se dispõem de técnicas convencionais viáveis industrialmente para sua obtenção, exceto pela polimerização em emulsão semeada, mas que também se limita a determinados tipos de reagentes (Ito et al., 2002). Desta forma, o aumento controlado do tamanho de partícula e/ou a manipulação da distribuição do tamanho de partículas de um látex polimérico previamente formado pode preencher viavelmente uma lacuna existente na produção de partículas poliméricas, além de criar novas possibilidades de aplicação de produtos obtidos através de certas especialidades, como os obtidos via processos em miniemulsão. 


\section{9 a 22 de outubro de 2014 \\ Florianópolis/SC}

Neste sentido, alguns trabalhos envolvendo o uso de processos de coagulação controlada para manipulação do tamanho e/ou da distribuição de tamanhos de partículas poliméricas tem sido apresentados (Ito et al., 2002; Kostansek, 2004; Cheng et al., 2004; Liu et al., 2014), porém, na maioria dos casos o agente coagulante utilizado para desencadear a coagulação é um eletrólito, geralmente íons $\mathrm{Ca}^{++}$, que pode levar à formação de aglomerados de baixa densidade e com forma irregular no regime mais comum, de agregação por difusão limitada (Diffusion limited aggregation DLA), além de adicionar mais um "contaminante" à formulação. Por outro lado, a diminuição da temperatura de transição vítrea $(T g)$ do polímero abaixo da temperatura do sistema sob condições específicas de operação pode levar à aglomeração e coalescência de partículas mantendo a forma esférica e a estabilidade coloidal do sistema. Adicionalmente, a incorporação de um solvente na matriz polimérica pode reduzir a $T g$ do polímero facilitando a superação da temperatura do meio, podendo ser facilmente removido e recuperado ao final do processo.

Segundo Klodt e Gougeon (2003), cada $1 \%$ em massa de pentano incorporado ao poliestireno (PS) leva à redução de até $7^{\circ} \mathrm{C}$ na $T g$ do polímero. Assim, o presente trabalho apresenta um estudo do emprego do pentano para promover a coagulação controlada de nanopartículas de PS com o objetivo de manipular o tamanho e distribuição de tamanho das mesmas mantendo a forma esférica. As nanopartículas foram obtidas previamente via polimerização em emulsão visando diferentes tamanhos e massas molares, os quais foram avaliados na etapa de coagulação juntamente com algumas condições de operação.

\section{EXPERIMENTAL}

\subsection{Materiais}

Nas polimerizações em emulsão foram utilizados estireno $(\mathrm{S})$, com grau de pureza superior a 99,6\% e concentração do inibidor p-terc-butilcatecol de 12 ppm, como monômero; terc-dodecanotiol (DDT) como agente de transferência de cadeia, lauril sulfato de sódio (SLS) como surfactante, bicarbonato de sódio como agente tamponante e persulfato de potássio (KPS) como iniciador. Todos os reagentes foram usados como recebidos e a água empregada como fase contínua foi destilada.

Na etapa de coagulação, as nanopartículas poliméricas (NPs), obtidas na etapa de polimerização, foram usadas como partículas precursoras. Pentano (mistura dos isômeros n- e iso-pentano), em grau técnico, foi utilizado como agente coagulante, e água destilada foi utilizada como diluente para manipulação do teor de sólidos.

\subsection{Procedimento}

As emulsões foram preparadas em um reator de vidro encamisado de $1000 \mathrm{~mL}$ a partir da dissolução do surfactante e do agente tamponante na água destilada, sob agitação mecânica de 400 rpm e fluxo de nitrogênio por $30 \mathrm{~min}$. Em seguida, foi adicionado o monômero ao meio reacional e, após 15 min, o iniciador previamente dissolvido. A adição de nitrogênio para a purga do oxigênio foi mantida durante toda a reação, que ocorreu a $70^{\circ} \mathrm{C}$ por 3 horas. Os detalhes de cada formulação, que apresentam teores de sólidos de apróximadamente 20\%, são mostrados na Tabela 1. 
Tabela 1 - Formulações utilizadas nas polimerizações em emulsão

\begin{tabular}{ccccccc}
\hline \multirow{2}{*}{ Reações } & \multicolumn{7}{c}{ Massa (g) } \\
\cline { 2 - 7 } & Água & SLS & NaHCO $_{3}$ & S & DDT & KPS \\
\hline NP1 & 600,00 & 1,620 & 0,14 & 144,00 & - & 1,60 \\
NP2 & 600,00 & 1,620 & 0,14 & 141,12 & 2,88 & 1,60 \\
NP3 & 600,00 & 0,610 & 0,14 & 144,00 & - & 1,60 \\
\hline
\end{tabular}

O processo de coagulação consistiu em transferir os componentes (látex, água e agente coagulante) para um tanque de aço de $200 \mathrm{~mL}$ e aquecer o mesmo até $120^{\circ} \mathrm{C}$ por $180 \mathrm{~min}$, contados a partir da estabilização da pressão interna. Em seguida, o tanque foi resfriado e a pressão do sistema foi reduzida através da abertura da válvula de alívio, possibilitando a recuperação do agente coagulante. $\mathrm{O}$ teor de sólidos usado em todos os ensaios de coagulação foi de $0,5 \%$ em relação à massa total, e na Tabela 2 são apresentados os valores do teor de pentano e da temperatura do sistema durante o alívio de pressão para cada condição avaliada.

Tabela 2 - Valor das variáveis alteradas nos testes de coagulação

\begin{tabular}{cccc}
\hline Testes & C1 & C2 & C3 \\
\hline Temperatura durante o alívio de pressão $\left({ }^{\circ} \mathrm{C}\right)$ & 99 & 99 & 80 \\
Teor de pentano $(\%$ relativo à massa de polímero) & 160 & 400 & 400 \\
\hline
\end{tabular}

\subsection{Caracterização}

A conversão dos reagentes $(X)$ durante as polimerizações foi determinada por análise gravimétrica. A distribuição de tamanho em intensidade $(P S D)$ e o diâmetro médio em z $(D p)$ das partículas foram determinados por espalhamento dinâmico de luz (Dynamic Light Scattering - DLS, Nanosizer Nano S ZEN1600, Malvern Intruments). A distribuição das massas molares e suas médias, ponderal e numérica, foram determinadas através da técnica de cromatografia por permeação em gel (Gel Permeation Chromatography - GPC, HPLC LC-20A, Shimadzu). A temperatura de transição vítrea $(T g)$ das amostras foi determinada através de análises de calorimetria diferencial de varredura (Differencial scanning calorimetry - DSC, Perkin Elmer, Jade DSC). A forma e a morfologia das partículas foram determinadas por microscopia eletrônica de transmissão (MET - JEM-1011, JEOL) e por microscopia eletrônica de varredura de alta resolução com canhão de emissão de elétrons por efeito de campo (MEV-FEG - JEOL JSM-6701F). Nesta última, as partículas foram imobilizadas em rezina e posteriormente removidas durante o corte, permanecendo apenas as cavidades deixadas pelas partículas na rezina.

\section{RESULTADOS E DISCUSSÃO}

\subsection{Etapa de Polimerização}

As reações de polimerização em emulsão foram realizadas visando à obtenção de nanopartículas 


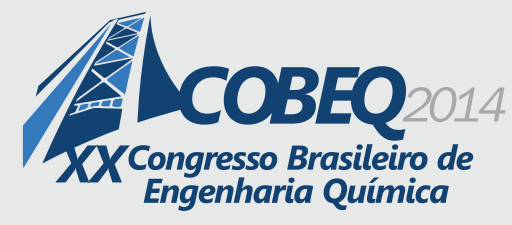

19 a 22 de outubro de 2014
Florianópolis/SC

de PS com diferentes diâmetros e massas molares para posterior comparação na etapa de coagulação. $\mathrm{Na}$ Tabela 3 são apresentados os principais resultados obtidos e na Figura 1 as imagens de MET correspondentes.

Tabela 3 - Resultados obtidos nas polimerizações em emulsão do estireno

\begin{tabular}{ccccccc}
\hline \multirow{2}{*}{ Reações } & \multirow{2}{*}{$\mathbf{X}(\boldsymbol{\%})$} & \multirow{2}{*}{$\mathbf{D}(\mathbf{n m})$} & \multirow{2}{*}{$\mathbf{T g}\left({ }^{\circ} \mathbf{C}\right)$} & \multicolumn{3}{c}{ Massas molares média $(\mathbf{g} / \mathbf{m o l})$} \\
\cline { 5 - 7 } & & & $\mathbf{M w} \times \mathbf{1 0}^{\mathbf{3}}$ & $\mathbf{M n} \times \mathbf{1 0}^{\mathbf{3}}$ & $\mathbf{M w} / \mathbf{M n}$ \\
\hline NP1 & 100 & 61 & 117 & 871,4 & 136,9 & 3,36 \\
NP2 & 99 & 66 & 107 & 33,6 & 9,4 & 3,57 \\
NP3 & 97 & 241 & - & 447,7 & 71,4 & 6,26 \\
\hline
\end{tabular}

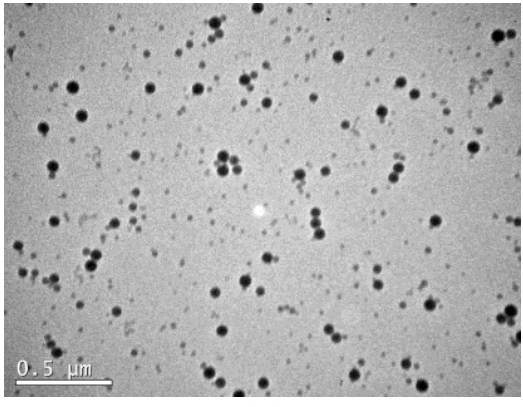

(a)

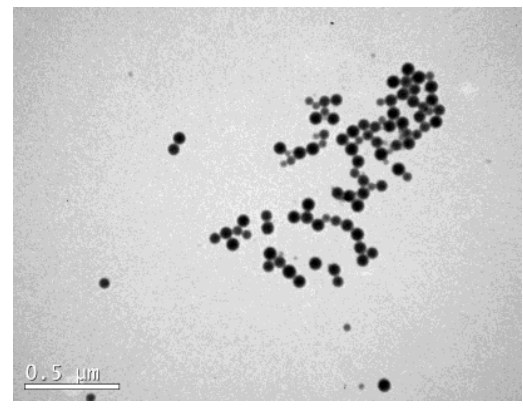

(b)

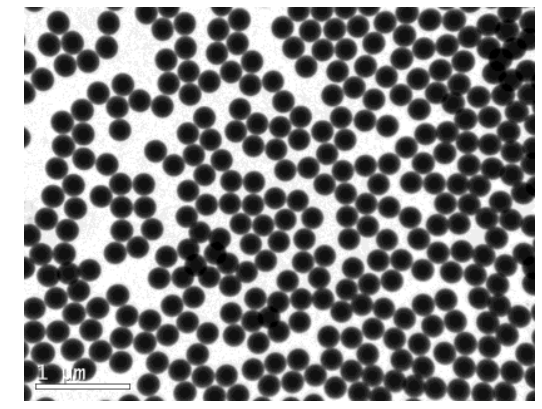

(c)

Figura 1 - Imagens de MET das nanopartículas de PS das reações NP1 (a), NP2 (b) e NP3 (c).

De acordo com os resultados, foi possível obter um aumento de aproximadamente 60 vezes no volume das partículas da reação NP3 em relação à NP1 através da redução da quantidade de surfactante no sistema, resultando ainda em uma distribuição monodispersa, como pode ser visto na Figura 1(c). Adicionalmente, o aumento do tamanho das partículas leva à diminuição do número de partículas $(N p)$, visto que a quantidade da fase dispersa é igual em todos os casos, e consequentemente, à diminuição da velocidade de reação, que é diretamente proporcional ao $\mathrm{Np}$; e da massa molar, uma vez que o aumento da frequência de entrada de radicais nas partículas contribui para o aumento das reações de terminação. Na reação NP2, a presença de agente de transferência de cadeia possibilitou a diminuição da massa molar em aproximadamente 25 vezes em relação à NP1, e da $T g$ do polímero como uma consequência direta da diminuição da massa molar (Billmeyer, 1984).

\subsection{Etapa de Coagulação}

As nanopartículas obtidas na etapa de polimerização foram submetidas a um tratamento térmico na presença de um leve solvente do polímero visando à coagulação controlada das mesmas. Primeiramente, as nanopartículas obtidas na reação NP1 foram submetidas a testes de coagulação com diferentes teores de pentano e temperatura durante o alívio de pressão ao final do processo (NP1-C1, NP1-C2 e NP1-C3), conforme a configuração mostrada na Tabela 2. Em seguida, a condição C2 foi empregada nos testes de coagulação das nanopartículas NP2 e NP3 (NP2-C2 e NP3-C2). Os resultados de $P S D$ das partículas antes e depois de passarem pelos testes de coagulação são mostrados na Figura 


\section{9 a 22 de outubro de 2014 \\ Florianópolis/SC}

2, sendo que, nas condições avaliadas, todos os látices apresentaram estabilidade coloidal após a etapa de coagulação. As imagens de MET e MEV-FEG das partículas que apresentaram aumento de tamanho significativo são apresentadas na Figura 3.

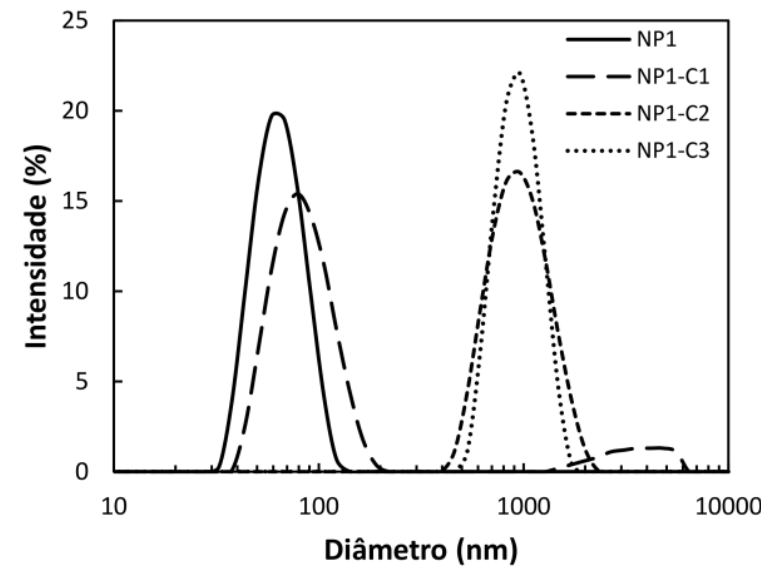

(a)

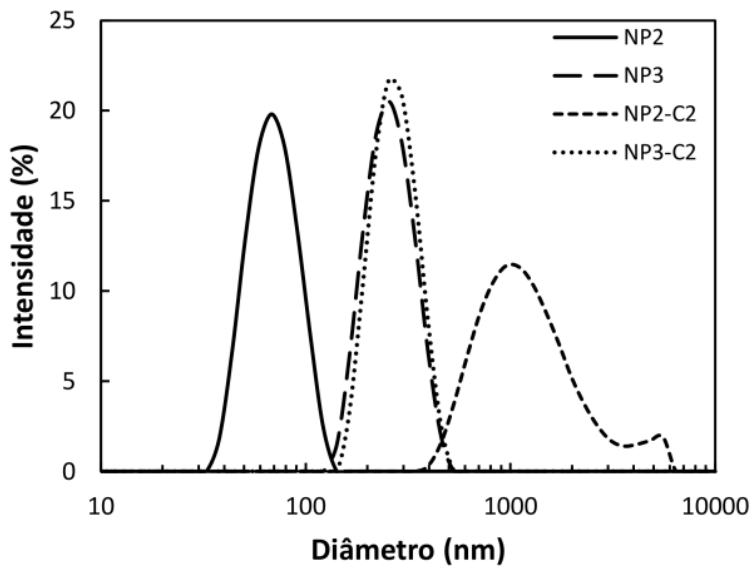

(b)

Figura 2 - PSD das partículas antes e depois dos testes de coagulação. (a) NP1; (b) NP2 e NP3.

Na Figura 2(a) pode ser vista a PSD para NP1 após diferentes condições de coagulação. Na avaliação da concentração de pentano (NP1-C1 e NP1-C2), pode-se observar que para o caso com concentração menor, $160 \%$, o processo resultou no surgimento de duas populações de partículas, sendo a principal caracterizada por um pequeno deslocamento na distribuição em relação às partículas percursoras e a segunda por um grande aumento de tamanho, sugerindo inclusive partículas com tamanhos acima do limite de leitura do DLS $(6,0 \mu \mathrm{m})$; porém, vale lembrar que a PSD em intensidade é mais sensível (sobrestima) às partículas maiores. Quando a concentração de pentano foi dobrada, passando para 400\%, observou-se apenas uma população de partículas esféricas com $D p$ de $895 \mathrm{~nm}$ e baixa polidispersidade, conforme pode ser visto na imagem de MET (Figura 3(b)). Como o fator considerado mais importante para promover a coalescência nesta abordagem consiste em operar o sistema a uma temperatura acima da $T g$ do polímero, a quantidade de pentano no sistema deve ser suficiente para saturar as fases aquosa e gasosa, e para se distribuir na forma de gotas na fase aquosa e, principalmente, nos aglomerados em crescimento. Assim, ao longo do processo, as cadeias poliméricas e as moléculas de surfactante presente nesses aglomerados podem rearranjar-se minimizando a energia livre do sistema e resultando em formas esféricas. Durante o processo de resfriamento, feito a temperatura ambiente, a solubilidade do pentano na matriz polimérica é reduzida dando início à migração do pentano para fora dos aglomerados resultando em partículas esféricas e homogêneas.

Ainda na Figura 2(a), o resultado de PSD obtido para as partículas NP1 submetidas ao teste de coagulação com $400 \%$ de pentano e despressurização a temperatura de $80^{\circ} \mathrm{C}$ (NP1-C3) mostrou alta similaridade com o resultado obtido pelo caso NP1-C2 (fato corroborado pela imagem de MET, Figura 3(c)), indicando que quando o sistema atinge $100^{\circ} \mathrm{C}$ durante o resfriamento, a $T g$ do polímero NP1 já se encontra acima desta temperatura, de modo que a temperatura utilizada durante a recuperação do 
pentano não afeta o tamanho e/ou forma das partículas finais obtidas.

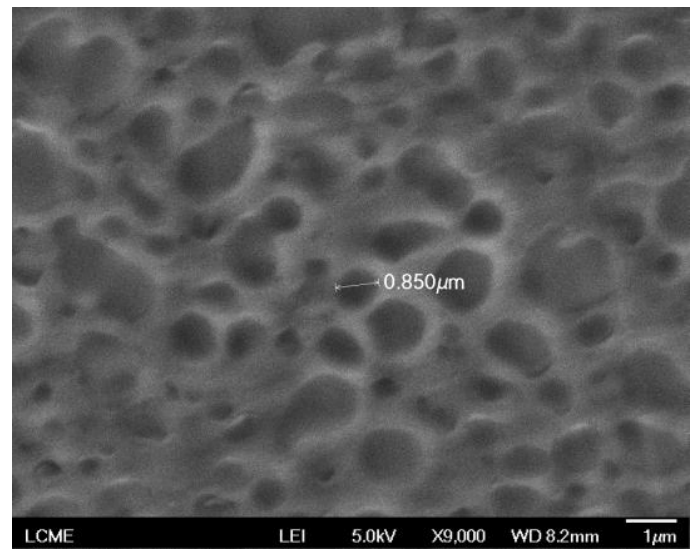

(a)

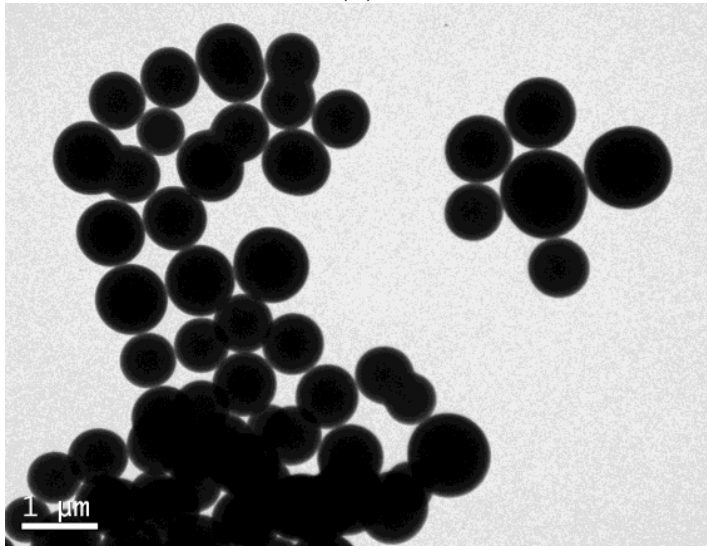

(c)

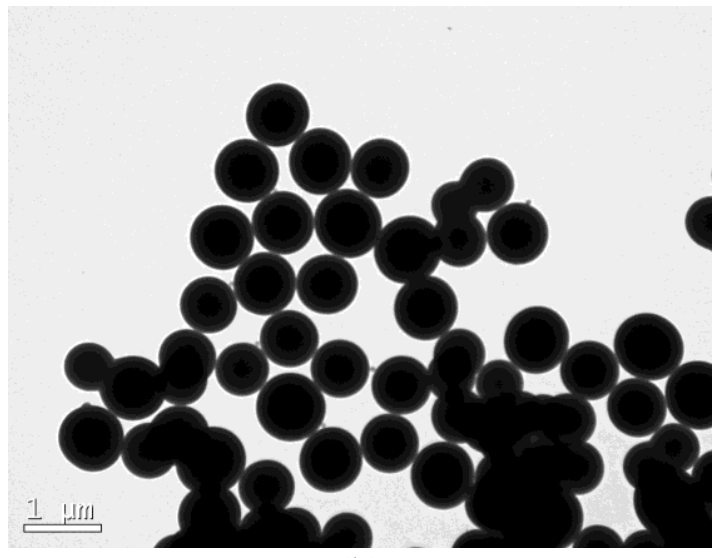

(b)

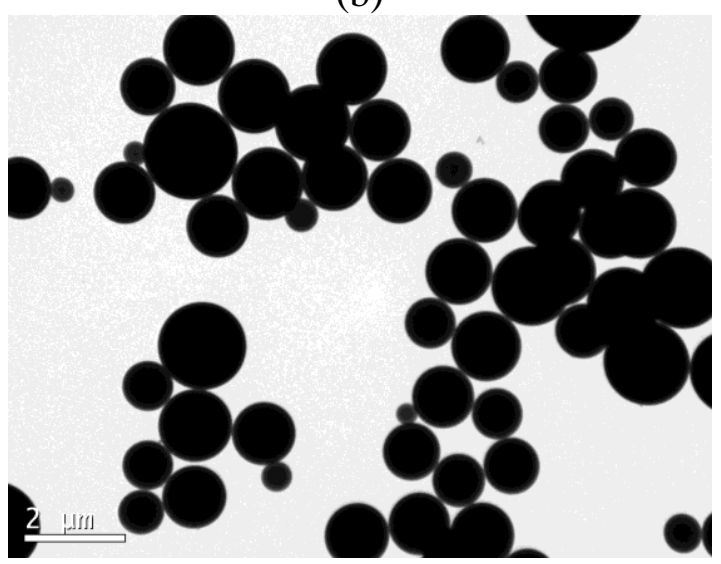

(d)

Figura 3 - Imagens de microscopia das partículas pós coagulação. (a) MEV-FEG NP1-C2; (b) MET NP1-C2; (b) MET NP1-C3; (c) MET NP2-C2.

Os resultados obtidos a partir da avaliação de diferentes partículas submetidas ao processo de coagulação, apresentados na Figura 2(b), mostram a importância do tamanho das partículas percussoras, ou seja, da área superficial total do sistema; e da massa molar do polímero, que reflete na $T g$ do mesmo. Para as partículas NP2-C2, a PSD mostra o surgimento de uma população de partículas ainda maiores que no caso NP1-C2, com $D p$ de $1090 \mathrm{~nm}$, alta polidispersidade e a indicação da presença de uma segunda população de partículas iniciando próximo dos 3,0 $\mu \mathrm{m}$, porém, como mencionado anteriormente, a quantidade de partículas maiores pode ser sobrestimada nas medidas de DLS, sendo que não foi possível observar partículas maiores deste valor através das imagens de MET. De qualquer forma, a diferença no tamanho final das partículas entre estes dois casos sugere que o processo de coagulação se estende por um período maior no caso NP2-C2, o que é esperado uma vez que a diferença na $T g$ é de $10^{\circ} \mathrm{C}$ e a área superficial disponível no sistema é aproximadamente igual, assim, nas mesmas condições de teor de pentano, temperatura e pressão, a $T g$ do polímero NP2 é inferior à do NP1. 


\section{9 a 22 de outubro de 2014 \\ Florianópolis/SC}

O caso NP3-C2, no qual as partículas precursoras apresentavam $D p$ de $241 \mathrm{~nm}$, não resultou em um aumento de partícula significativo, com $D p$ final de $263 \mathrm{~nm}$ indicando que praticamente não houve coagulação das mesmas. Para essas partículas, a área superficial inicial disponível no sistema é quase 4 vezes menor que para as partículas NP1, o que torna a quantidade de pentano difundida na matriz polimérica muito menor, e consequentemente, a redução da Tg. Assim, a temperatura do sistema não excede a $T g$ o suficiente para provocar o amolecimento do polímero necessário para resultar na coagulação das partículas após a colisão das mesmas.

\section{CONCLUSÕES}

Nanopartículas de poliestireno com diferentes tamanhos e massas molares, obtidas via polimerização em emulsão, foram submetidas a ensaios de coagulação promovida pela presença de um alcano de baixa massa molar. Os resultados mostraram o aumento do tamanho das partículas de até 4500 vezes o volume inicial (considerando o $D p$ obtido via DLS para NP2-C2) quando pentano foi usando em grande quantidade juntamente com partículas precursoras de diâmetro inferior a $100 \mathrm{~nm}$, indicando que a formação dos aglomerados deve ocorrer devido ao inchamento rápido das partículas com pentano e/ou através da colisão das partículas precursoras nas gotas de pentano. Assim, as partículas plastificadas combinadas com a redução da área recoberta pelo surfactante após o inchamento origina clusters esféricos de poliestireno-pentano com baixa $T g$ altamente suscetíveis à coalescência mediante colisão com outras partículas/aglomerados. Durante o resfriamento lento, a solubilidade do pentano na matriz polimérica diminui ocorrendo a difusão deste para fora do aglomerado, intensificando ainda mais a diminuição da solubilidade visto que sua saída possibilita o aumento da $\mathrm{Tg}$. Abaixo de $100^{\circ} \mathrm{C}$, o alívio de pressão pode ser realizado em qualquer temperatura possibilitando a recuperação total do pentano sem comprometer a estabilidade do sistema.

\section{AGRADECIMENTOS}

Os autores agradecem o Conselho Nacional de Desenvolvimento Científico e Tecnológico (CNPq), pelo suporte financeiro, o Laboratório Central de Microscopia Eletrônica (LCME) da Universidade Federal de Santa Catarina (UFSC), pelas análises de MET, e o Laboratório de Propriedades Físicas de Alimentos (PROFI) da UFSC, pelas análises de Tg.

\section{REFERENCIAS}

BILLMEYER, F. W. Textbook of Polymer Science. $3^{\text {a }}$ ed. New York: John Willey \& Soons, 1984.

CHENG, H.; WU, C.; WINNIK, M. A. Kinetics of Reversible Aggregation of Soft Polymeric Particles in Dilute Dispersion. Macromolecules, v. 37, p. 5127-5129, 2004.

ITO, F.; MA, G.; NAGAI, M.; OMI, S. Study of Particle Growth by Seeded Emulsion Polymerization Accompanied by Electrostatic Coagulation. Colloids and Surfaces A: Physicochem. Eng. Aspects, v. 201, p. 131-142, 2002.

KLODT, R. -D.; GOUGEON, B. Particle Foam Based on Expandable Polystyrene (EPS). In: Modern Styrenic Polymers: Polystyrenes and Styrenic Copolymers. New York: John Wiley \& Sons, 2003. 
KOSTANSEK, E. Controlled Coagulation of Emulsion Polymers. JCT Research, v. 1, p. 41-44, 2004.

LIU, B.; ZHANG, M.; GUI, Y.; CHEN, D.; ZHANG, H. Effect of aqueous phase composition on particle coagulation behavior in batch emulsion polymerization of styrene. Colloids and Surfaces A: Physicochem. Eng. Aspects, v. 452, p. 159-164, 2014. 